\title{
Recent advances in ceramic implants as drug delivery systems for biomedical applications
}

\author{
Montserrat Colilla \\ Miguel Manzano \\ María Vallet-Regí \\ Departamento de Química Inorgánica \\ y Bioinorgánica, Facultad de Farmacia, \\ Universidad Complutense de Madrid, \\ Madrid, Spain; Centro de Investigación \\ Biomédica en Red, Bioingeniería, \\ Biomateriales y Nanomedicina, \\ CIBER-BBN, Spain
}

\begin{abstract}
Research in the development of new bioceramics with local drug delivery capability for bone regeneration technologies is receiving great interest by the scientific biomedical community. Among bioceramics, silica-based ordered mesoporous materials are excellent candidates as bone implants due to two main reasons: first, the bioactive behavior of such materials in contact with simulated body fluids, ie, a carbonate hydroxyapatite similar to the mineral phase of bone is formed onto the materials surfaces. Second, their capability of acting as delivery systems of a large variety of biologically active molecules, including drugs to treat bone infection, inflammation or diseases, and molecules that promote bone tissue regeneration, such as peptides, proteins, growth factors, and other osteogenic agents. The recent chemical and technological advances in the nanometer scale has allowed the design of mesoporous silicas with tailored structural and textural properties aimed at achieving a better control over molecule loading and release kinetics. Moreover organic modification of mesoporous silica walls has been revealed as a key strategy to modulate molecule adsorption and delivery rates.
\end{abstract}

Keywords: silica-based ordered mesoporous materials, controlled delivery systems, bone tissue regeneration

\section{Introduction}

The biomedical community has traditionally used two types of ceramic implants for bone tissue regeneration technologies: inert materials such as alumina, zirconia, and carbon (Hulber 1993; Wise et al 1995) and bioactive ceramics (Vallet-Regí 2001a), which interact with the physiological environment when implanted leading to their integration in the living tissue (Hench 1984; Kokubo et al 1990; Langer et al 1993; Hench 1998). Depending on the patient needs, an appropriate ceramic material should be selected to manufacture a determined implant. When hard tissues, natural composite materials, are aimed, the synthetic approach of these composite materials is a way to mimic Nature in the laboratory (Vallet-Regí et al 2006a). In the past few years bioactive ceramics like calcium phosphates (Yamamuro et al 1990a), bioactive glasses and glass-ceramics (Yamamuro et al 1990b) have been deeply investigated. Organic-inorganic hybrid materials have been also widely proposed as good candidates in the biomaterials field because they combine the properties of ceramics and organic polymers on the nanometric scale (Tsuru et al 1997; Chen et al 1999; Sanchez et al 2005; Colilla et al 2006; Vallet-Regí et al 2006a). Star gels have been recently reported as alternative materials for bone tissue regeneration because they combine the good mechanical properties of certain organic-inorganic hybrids with the bioactivity of conventional glasses (Manzano et al 2006; Vallet-Regí et al 2006b). However, much research effort has been committed to the investigation of ordered mesoporous silica materials in the biomedical field for two main reasons: their capability to regenerate bone tissue (Vallet-Regí et al 2006c, 2006d) combined with their drug delivery possibilities (Vallet-Regí et al 2006e, 2007a). 
When these silica-based ordered mesoporous materials are exposed to the physiological environment, a series of chemical reactions take place in the material-living tissue interface, which lead to the material incorporation into the living tissue. Those processes are confirmed through the nucleation and growth on the bioceramic surface of a layer of carbonated hydroxyapatite which is analogous to the mineral phase of bone tissues (Vallet-Regí et al 2004a). Thus, this type of bioceramics would contribute to bone tissue regeneration. Recently, much research effort has been devoted to the design of mesoporous matrices with appropriated structural, textural and chemical properties in order to accelerate the formation of the apatite-like layer (IzquierdoBarba et al 2005a; Vallet-Regí et al 2005).

The open texture and outstanding textural properties of mesoporous materials have inspired the usage of this type of materials as controlled delivery systems of biologically active molecules (Vallet-Regí et al 2001b). In fact, their high pore volume permits to host a large amount of a determined biologically active molecule. Also their ordered pore network allows a fine control of the molecule load and release kinetics. Since molecules adsorption into the mesopores is a surface phenomenon, their high surface area will also favor the adsorption of a large amount of molecules into their mesopores. And the last, but no the least, is the possibility of functionalizing the silanol-containing surface with a selected organic group depending on the molecule to be adsorbed to allow a better control over molecule loading and release.

This double perspective of the mesoporous materials, tissue regeneration and drug delivery, has promoted the research of these materials for biomedical applications in the last few years (Vallet-Regí et al 2008; Izquierdo-Barba et al 2008). In fact, the outstanding properties of silica-based ordered mesoporous materials make them suitable to be used as starting materials for the further design of scaffolds for bone tissue engineering. The regular repeating mesoporous structures of these silica-based mesoporous materials has motivated the adsorption in their pores and subsequent release of a large variety of biologically active species such as proteins, polypeptides or amino acids. (Yiu et al 2001a; Deere et al 2002; Balas et al 2008). Within their possible biomedical applications, the case of protein delivery systems using serum albumins should be highlighted (Vallet-Regí et al 2007b) because these proteins represent one of the major components in plasma proteins in humans. Albumin is usually composed of a single-chain of 582 amino acids with an average length of $10 \mathrm{~nm}$ and width of $6 \mathrm{~nm}$ (Peters 1995; Sugio et al 1999; RCSB 2008). Besides contributing to colloid osmotic blood pressure and to the maintenance of the blood $\mathrm{pH}$, the most outstanding property of albumin is its ability to bind reversibly an incredible variety of ligands. As a consequence, this multifunctional transport protein can be used as carrier or reservoir of certain polypeptides with special interest in osseous regeneration technologies. Therefore, albumin could store, protect, and deliver determined peptides useful for bone regeneration to specific places. Moreover, albumins are also capable of binding a wide variety of drugs than can be delivered to sites of pharmacological action (Carter et al 1994). Several proteins have been demonstrated to retain their functionality without being denatured inside the frameworks of silica-based mesoporous materials (Deere et al 2002; Cheng et al 2003; Lei et al 2004; Vinu et al 2004; Hartmann 2005; Katiyar et al 2005; Lee et al 2006; Slowing et al 2007). These findings open up many expectations for the research in the adsorption of proteins and other biologically active agents into mesoporous silicas.

The adsorption and release characteristics of each porous material depend on the physicochemical properties of the individual material. Among those properties, it should be highlighted the pore diameter, surface area, and pore volume, which are critically reviewed here. To achieve this task, it is necessary to look into the synthesis conditions of these materials, which modulate the physicochemical properties of each structure.

\section{Silica-based ordered mesoporous materials: General remarks}

Silica-based ordered mesoporous materials are synthesized using a surfactant templating method (Figure 1) and present ordered porous structures with narrow pore size distributions and some of them with thick walls, which enhance their stability. They also show large surface areas and large pore volumes. In the early 1990s, Japanese researchers (Yanagisawa et al 1990; Inagaki et al 1993) and Mobil Corporation research and development scientists (Kresge et al 1992) for the first time reported the synthesis of novel periodic mesostructured materials, known as KSW-n and M41S family, respectively. These materials are characterized by regular arrays of uniform channels, whose dimensions can be tailored through the choice of surfactants, additives and synthesis conditions in the so-called liquid crystal templating mechanism (Beck et al 1992). This process is based on the formation of liquid crystals in mixtures of polar solvents and surfactants with a non-polar tail group. The formation of the liquid crystals is as follows: an increasing amount of surfactant molecules is dissolved in an aqueous solution, 


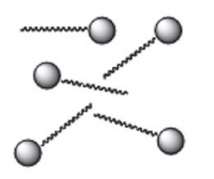

Surfactant molecules
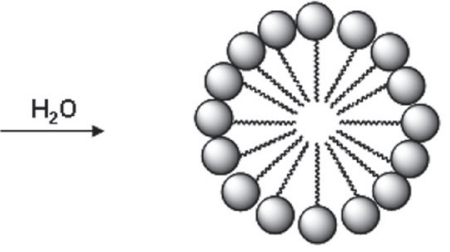

Surfactant micelle
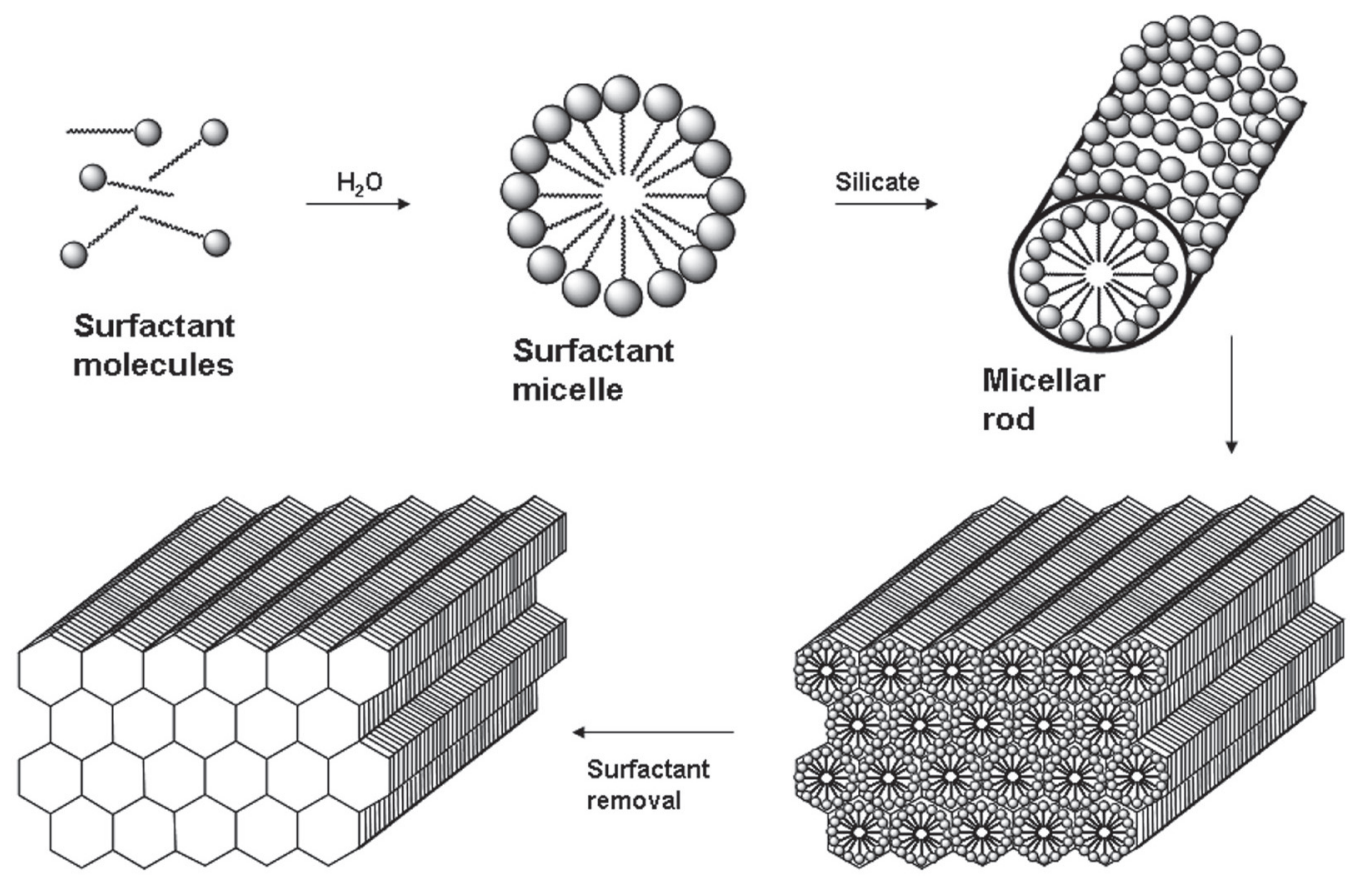

Mesoporous material

Figure I Schematic representation of liquid crystal mechanism used to describe the synthesis of silica-based ordered mesoporous materials.

and when surfactant concentration reaches the critical micellar concentration $(\mathrm{cmc})$, the surfactant molecules cluster together as micelles. These micelles are formed because the hydrophobic tails of the surfactant tend to congregate while their hydrophilic heads are procuring protection in water (Figure 1). The final mesostructure of the material will depend on the organization of the surfactant molecules into the micellar liquid crystals which act as templates for the formation of the mesoporous materials. These liquid crystal structures depend on the composition and chemical nature of the surfactant, and also on the solution mixture conditions, such as surfactant concentration, $\mathrm{pH}$, temperature, the presence of additives, etc. In the final step of the synthetic process, once the silica source has condensed around the micelles, the surfactant is removed by thermal degradation or solvent extraction. This surfactant removal gives rise to a network of cavities within the silica framework that governs the physicochemical properties of the material. Different mesoporous structures have been developed in the last few years as a consequence of different modifications in the synthetic procedure. Among them, the most representative and employed materials are MCM-n (Mobil Composition of Matter) series (Beck et al 1992; Kresge et al 1992; Firouzi et al 1997; Zhang et al 1997; Kruk et al 2001; Kaneda et al 2002), SBA-n (Santa BArbara materials) series
(Zhao et al 1998; Ravikovitch et al 2002a, 2002b), MSU-n (Michigan State University) series (Bagshaw et al 1995), KIT-n (Korean Advanced Institute of Science and Technology) series (Ryoo et al 1996), FSM-n (Folded Sheet Material) series (Inagaki et al 1996), and AMS-n (Anionic Surfactant templated Mesoporous silica) series (Che et al 2003).

In general, silica-based ordered mesoporous materials display exceptional properties, such as stable mesoporous structure, high surface area (ca $1000 \mathrm{~m}^{2} / \mathrm{g}$ ), large pore

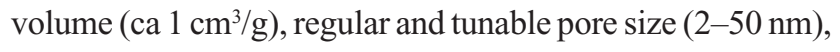
homogeneous pore morphology, non-toxic and biocompatible behavior, and the possibility of undergoing chemical functionalization of pore walls with different organic groups. In fact, these inorganic mesoporous materials offer the possibility of tuning the chemical properties of their surfaces to achieve the desired properties (Hoffmann et al 2006). Two major methods of producing functionalized materials have been widely used: direct functionalization (Antochshuk et al 2000), which involves the addition of a trialkoxysilane with the selected functional group to the reaction mixture during the synthesis process; and post-synthesis functionalization (Liu et al 1998), which involves the grafting of the functional group onto the mesoporous material after surfactant removal. The direct functionalization method, also known as the co-condensation process, presents some advantages like 
a homogeneous dispersion of the functional group, retention of the width of the pores of the original framework, and the presence of available silanol groups for the adsorbates. However, there are some drawbacks like the possibility of absence of the functional groups on the surface of the material, the possible loss of structure and order of the final structure, and the need of removing the surfactant by solvent extraction rather than by calcination, which could lead to an incomplete removal of the surfactant molecules. On the other hand, the post-synthesis functionalization method presents the advantages of retention of the order and structure, and the sureness that the functional groups will be located on the surface of the pore walls. Though this pathway means an extra step in the synthetic process and a reduction in the pore diameter, and also it might present a heterogeneous distribution of the grafted groups, it brings a noticeable change in the adsorption characteristics of the silica surface as well as in its polarity. The chemical modification of the pore walls would be selected depending on the molecule to be adsorbed, taking into account the desired load and release kinetics.

\section{Tailoring the performance of mesoporous controlled delivery systems}

Since 2001, when MCM-41 was proposed for the first time as controlled delivery system (Vallet-Regí et al 2001b), different mesoporous matrices have been tested as delivery systems of several drugs, such as ibuprofen (Muñoz et al 2003; Izquierdo-Barba et al 2005b; Manzano et al 2008), gentamicin (Xue et al 2004; Doadrio et al 2006), amoxicillin (Vallet-Regí et al 2004b), erythromycin (Izquierdo-Barba et al 2005b; Doadrio et al 2006), vancomycin (Yang et al 2005), naproxen (Cavallaro et al 2004), aspirin (Zeng et al 2005; Zhu et al 2005), diflunisal (Yang et al 2005), captopril (Qu et al 2006), and alendronate (Balas et al 2006; Nieto et al 2008). Moreover, mesoporous silicas have been also evaluated as carriers of other biologically active species, such as proteins, eg, bovine serum albumin [BSA] (Song et al 2005; Vallet-Regí et al 2007b) and certain amino acids, eg, $L$-Tryptophan ( $L$-Trp) (Balas et al 2008).

The textural properties of silica-based ordered mesoporous materials seem to govern the loading and release of biologically active molecules. Therefore, the influence of pore diameter, surface area, and pore volume in molecule adsorption and delivery kinetics will be tackled and critically discussed in the following sections. Moreover, organic modification of silica walls with different functional groups to achieve a better control over molecule loading and release rate will be also described.

\section{Pore diameter: An adsorptive size selectivity factor and release rate modulator}

The adsorption of molecules into silica-based ordered mesoporous materials is a size-selective process and consequently, the mesopore diameter determines the size of the molecule to be hosted. That is, if the molecule is smaller in size than the pore opening, it would have access to the large internal surface area and mesoporous volume of the material. On the contrary, if not, it would adsorb only on the external surface of the material. Usually, the molecule-loading process is carried out by soaking the mesoporous carrier in a highly concentrated molecule solution. Hence, the adsorptive properties of mesoporous materials will determine the amount of adsorbate loaded. Therefore, considering that the size of common drugs falls into the nanometer scale and that mesopore diameters can be tuned from $1.5 \mathrm{~nm}$ to several tens of nanometers, it seems reasonable to think that mesoporous matrices are able to host a wide range of biologically active molecules, ranging from small drugs to macromolecules such as large-size proteins.

Pore diameter has been probed to act as a drug-release rate modulator. When MCM-41 was reported for the first time as a drug delivery system (Vallet-Regí et al 2001b), several cationic surfactants with different chain lengths were employed, which led to MCM-41 materials with different pore sizes. Thus, the surfactant with the longest chain led to the MCM-41 with the largest pore diameter. Ibuprofen was chosen as the model drug and drug loading and release studies were carried out. Loading tests showed that the MCM-41 with the largest pore size released $68 \%$ of the loaded drug after $24 \mathrm{~h}$ into a simulated body fluid. On the contrary, the MCM-41 with the smallest pore diameter released only $55 \%$ of the loaded drug after the same time period. Further studies confirmed the influence of pore size on drug delivery rate (Horcajada et al 2004). In this research, cationic surfactants with hydrocarbon chains of different lengths were used as structure directing agents, leading to MCM-41 materials with diverse mesopore diameters, ranging from $2.5 \mathrm{~nm}$ (short-chain surfactants) to $3.6 \mathrm{~nm}$ (long-chain surfactants), as displayed in Table 1. The resulting release parameters, which are summarized in Table 1, revealed that the greater the mesopore diameter, the larger the amount of ibuprofen released.

Pore diameter is a limiting factor in molecule adsorption when the confinement of really large molecules, such as 
Table I Mesopore diameters of MCM-4I matrices obtained using surfactants with different chain lengths and ibuprofen release after 24 hours of assay. After 48 hours of test, all ibuprofen was released from $C_{12} T A B$ and $C_{16}$ TAB matrices; on the other hand, after 55 hours of assay, ibuprofen release was not completed for $85 \% \mathrm{C}_{8}$ TAB- $15 \% \mathrm{C}_{10}$ TAB and $70 \% \mathrm{C}_{8}$ TAB- $30 \% \mathrm{C}_{10}$ TAB

\begin{tabular}{lll}
\hline Surfactant* & $\begin{array}{l}\text { Pore diameter } \\
(\mathbf{n m})\end{array}$ & $\begin{array}{l}\text { lbuprofen released } \\
\text { after } \mathbf{2 4} \mathbf{h}(\%)\end{array}$ \\
\hline $85 \% \mathrm{C}_{8}$ TAB- $15 \% \mathrm{C}_{10}$ TAB & 2.5 & 41 \\
$70 \% \mathrm{C}_{8}$ TAB-30\% $\mathrm{C}_{10}$ TAB & 2.7 & 42 \\
$\mathrm{C}_{12}$ TAB & 3.3 & 49 \\
$\mathrm{C}_{16}$ TAB & 3.6 & 61 \\
\hline
\end{tabular}

Abbreviations: $C_{8} T A B$, octyltrimethylamonium bromide; $C_{10} T A B$, decyltrimethylamonium bromide; $C_{12} T A B$, dodecyltrimethylamonium bromide; $C_{16} T A B$, hexadecyltrimethylamonium bromide.

proteins and other biologically active molecules, is targeted. Therefore, the adsorption of globular proteins on mesoporous MCM-41 (with pore diameters ranging from 2 to $5 \mathrm{~nm}$ ) has been reported in the literature (Deere et al 2002). In general, proteins with hydrodynamic dimensions larger than the mesopore diameter were adsorbed on the outer surface of MCM-41 (Katiyar et al 2005). For this reason, when the confinement of proteins into mesoporous matrices is targeted, large pore mesoporous materials should be employed. The group of Prof. Wright (Yiu et al 2001b) investigated the influence of protein dimensions on the adsorption into SBA-15 mesoporous molecular sieve, whose silica walls were organically modified using thiol groups. For this purpose, a series of proteins with molecular weights ranging from 12000 to $76000 \mathrm{u}$ were used to investigate adsorption on SBA-15 materials. The structures of the employed proteins together with their dimensions in nanometers (RCSB 2008) are displayed in Figure 2. Further adsorption studies revealed that the proteins with the smallest sizes, ie, cytochrome c, lysozyme, myoglobin, and $\beta$-lactoglobulin, showed significant adsorption. On the contrary, the proteins with the largest sizes, ie, conalbumin, serum albumin and ovalbumin were excluded from the internal surfaces of thiol-functionalized SBA-15. This fact agrees with the sieving expected from the $5.1 \mathrm{~nm}$ pore size of thiol-functionalized SBA-15 when compared to the proteins dimensions. Moreover, the exclusion of ovalbumin $(4.0 \times 5.0 \times 7.0 \mathrm{~nm})$, with dimensions quite close to thiol-functionalized SBA-15 matrix, indicates that this size selectivity is rigorous and that there are very few

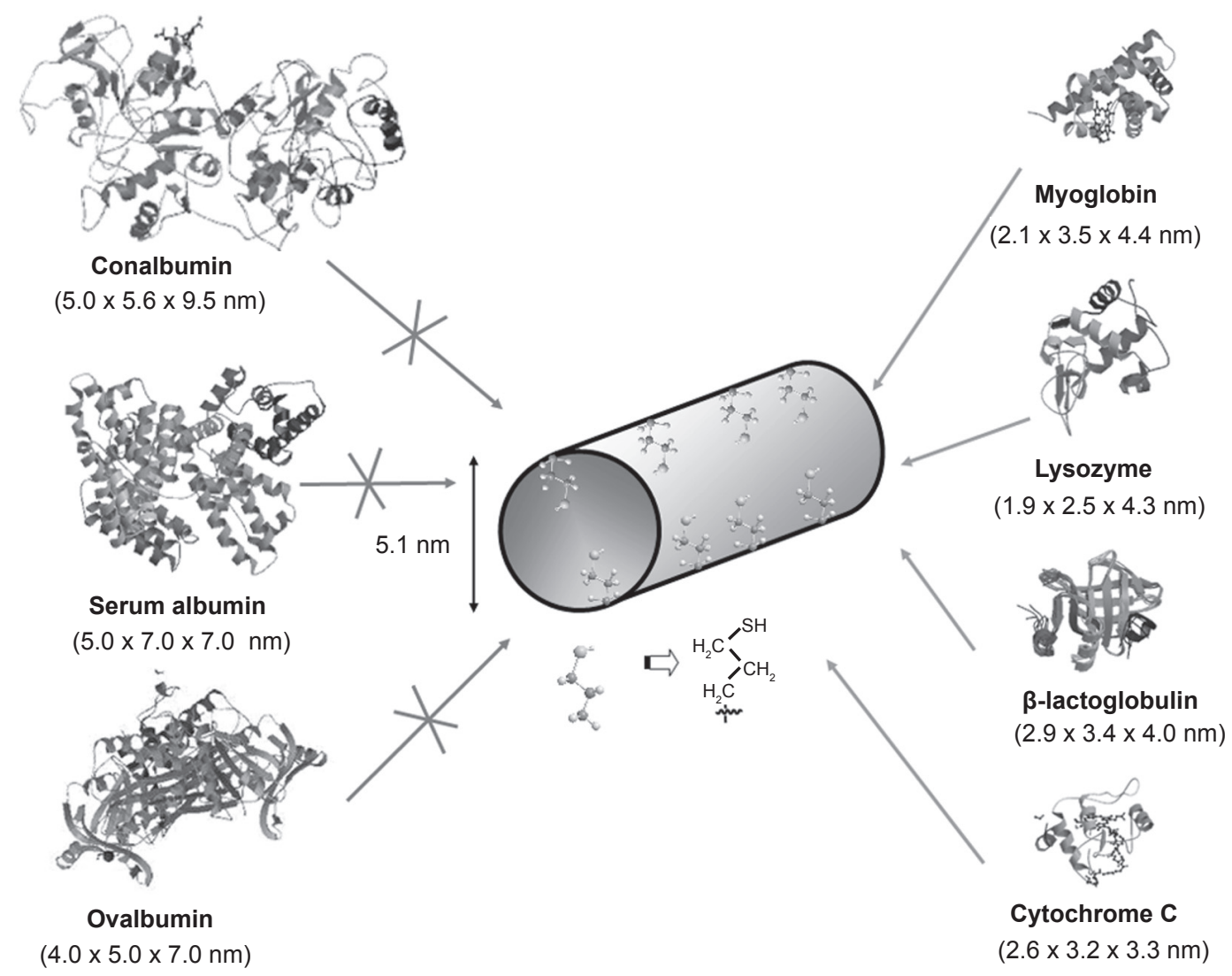

Figure 2 Schematic representation of the structure of different proteins with diverse dimensions (RCSB 2008) compared to thiol-functionalized SBA-I5. 
pores appreciably larger than the average pore size. It should be remarked that the maximum amounts of protein loaded, expressed as a volume percent of the available internal pore volume ( $5 \%$ for $\beta$-lactoglobulin, $15 \%$ for myoglobin, and $43 \%$ for cytochrome c) showed that the protein molecules were adsorbed within the mesopores and not only on the external surfaces.

From the above results it can be deduced that when the confinement of large size proteins is the aim, large pore mesoporous matrices are needed. This fact inspired the idea of using SBA-15 as a mesoporous carrier employing different hydrothermal treatments during the synthesis to wide the pore diameter of SBA-15 (Vallet-Regí et al 2007b). Hence, pore diameters ranging from $8.2 \mathrm{~nm}$ to $11.4 \mathrm{~nm}$ were obtained for SBA-15 materials submitted to hydrothermal treatments periods ranging from 1 to 7 days (Figure 3a). These pore dimensions seem suitable to host large-size proteins. Moreover, BSA loading dependence on SBA-15 diameter was observed, as displayed in Figure 3b. Hence, the amount of BSA loaded was $15 \%, 23 \%, 24 \%$, and $27 \%$ (weight percentage) for SBA-15 exhibiting pore diameters of $8.2,9.5,10.5$, and $11.4 \mathrm{~nm}$, respectively.

With the aim of promoting host-guest SBA-15-protein interaction, an organic modification of silica surface was performed using aminopropyl groups and following the post-synthesis method (Vallet-Regí et al 2007b). Thus, the amino groups of SBA-15 modified materials would undergo attracting electrostatic interactions with the carboxylic fraction of amide groups from the protein. As mentioned before, organic functionalization always leads to a decrease in the mesopore diameter. The BSA molecule is just on the limit of the mesopore dimensions, and thus, after aminofunctionalization the amount of BSA loaded decreased compared to unmodified matrices (see Table in Figure 3c). However, the BSA loading on amino-modified SBA-15 matrices underwent a behavior comparable to unmodified-matrices in terms of loading increment as the pore size increased.

The amino functionalization of SBA-15 had a noticeable influence on BSA delivery kinetics (Figure 3d). The BSA release from pure silica mesopore surfaces essentially showed a burst profile, where more than $90 \%$ of the adsorbed protein was released within the initial $24 \mathrm{~h}$ of tests. The rest of the adsorbed protein was linearly released up to complete delivery in $192 \mathrm{~h}$ in all tested materials, regardless of the hydrothermal treatment carried out for synthesis. However, amino-modified SBA-15 materials showed an incomplete release of the protein from the mesopores in all cases. This partial protein retention was attributed to the strong

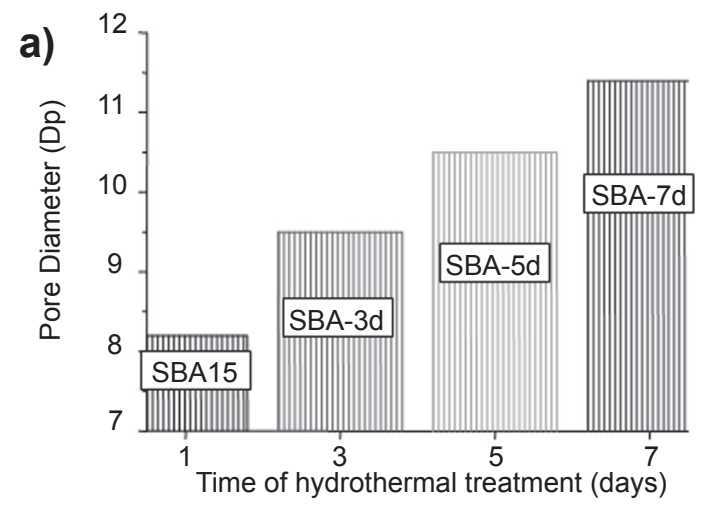

c)

\begin{tabular}{ccc}
\hline Sample & $\mathrm{D}_{\mathrm{mp}}(\mathrm{nm})$ & BSA loaded $(\%)$ \\
\hline SBA15-NH & 7.5 & 10.0 \\
SBA15-3d-NH & 8.4 & 15.3 \\
SBA15-5d-NH & 9.2 & 18.5 \\
SBA15-7d-NH & 9.8 & 28.5 \\
\hline
\end{tabular}
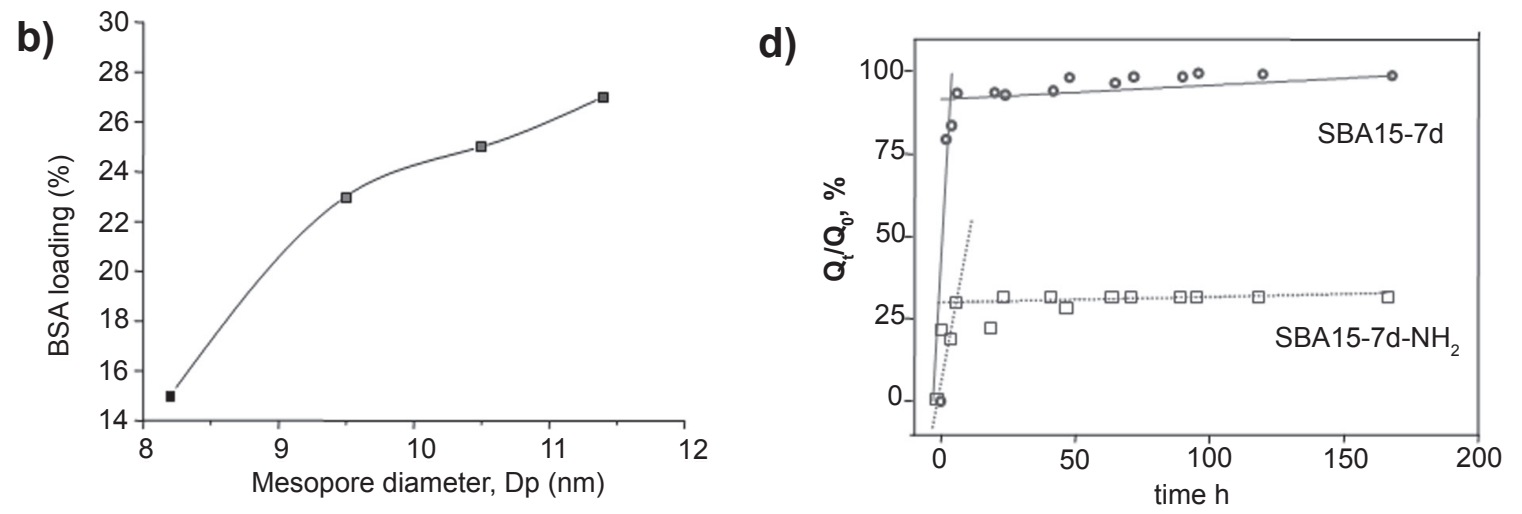

Figure 3 a) Effect of hydrothermal treatment on SBA- 15 mesopore diameter.b) BSA loading into SBA- 15 mesoporous matrices with different pore diameter. c) Table showing the BSA loading on functionalized matrices with different pore diameters. d) Release profiles of BSA from unmodified and amino-modified matrices. 
attracting interaction of silica walls amine groups with the protein. After $192 \mathrm{~h}$, the released protein ranged from $25 \%$ $\left(\mathrm{SBA}-15-7 \mathrm{~d}-\mathrm{NH}_{2}\right)$ up to $60 \%\left(\mathrm{SBA}-15-3 \mathrm{~d}-\mathrm{NH}_{2}\right)$ of the initially loaded amount of protein. As an illustrative example, Figure $3 \mathrm{~d}$ shows BSA release profiles of SBA-15 submitted to 7 days of hydrothermal treatment.

Therefore, the pore size has been revealed as a key factor governing the adsorption of proteins in mesoporous materials. The pore size should be noticeably larger than the protein to allow the diffusion of the protein into the mesopores. However, once the protein is adsorbed, the ruling factor on the release kinetics has been shown to be the organic functionalization, due to the host-guest attracting interactions.

\section{Influence of surface area}

The adsorption of biologically active molecules into mesoporous matrices is governed by the chemical interaction between silanol groups covering the silica surface and the functional groups of the guest molecule. Therefore, surface area is expected to determine the amount of molecules confined into the silica matrix. This fact was probed when MCM-41 mesoporous matrices exhibiting different surfaces areas, $\mathrm{S}_{\mathrm{BET}}$, ranging from 768 to $1157 \mathrm{~m}^{2} / \mathrm{g}$, which were synthesized using surfactants with diverse length-chains, were employed as ibuprofen delivery systems (Horcajada et al 2004). The amount of ibuprofen loaded was found to depend on the surface area, as graphically displayed in Figure 4. As expected, there was an increase in ibuprofen loading amount with the enlargement in the surface area values.

Surface area influence was also shown when alendronate, a potent bisphosphonate used in osteoporosis treatments, was

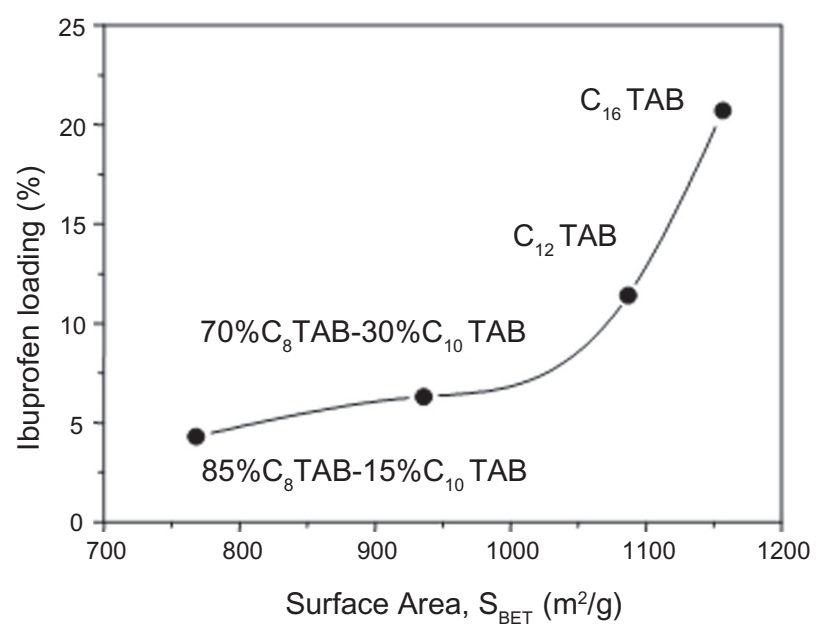

Figure 4 Dependence of ibuprofen loading on surface area of MCM-4I mesoporous materials synthesized using diverse cationic surfactants with different length-chains. The surfactants and the percentage employed are also displayed (See footnote on Table I). confined into MCM-41 and SBA-15 mesoporous matrices (Balas et al 2006). Both mesoporous matrices exhibited the same structure (2D hexagonal and $p 6 \mathrm{~mm}$ symmetry) but different surface areas, $\mathrm{S}_{\mathrm{BET}}$ of 1157 and $719 \mathrm{~m}^{2} / \mathrm{g}$ for MCM-41 and SBA-15, respectively. When both matrices were loaded with alendronate under the same conditions, the maximum amounts of drug loaded were $14 \%$ and $8 \%$ for MCM-41 and SBA-15, respectively. This fact showed the clear dependence of maximum drug load on the matrix surface area in agreement with results reported later in the literature (Qu et al 2006). MCM-41 and SBA-15 were functionalized using amino groups with the aim of increasing the attracting host-guest interactions, and alendronate loading and release studies were carried out (Balas et al 2006). The amount of alendronate loaded followed the same trend that unmodified materials, ie MCM-41- $\mathrm{NH}_{2}$ loaded more alendronate $(37 \%)$ than $\mathrm{SBA}-15-\mathrm{NH}_{2}(22 \%)$ as a result of the higher surface area of MCM-41 (Table 2). In addition, it should be noticed that the amount of alendronate loaded in modified materials was almost 3 times larger than those of unmodified materials. This fact can be explained by the stronger attracting interactions between phosphonate groups and amino groups of modified materials compared to the weaker interaction taking place between phosphonate groups and silanol groups from unmodified matrices (Figure 5). Regarding alendronate release, it should be highlighted that in all cases an initial burst effect was observed. This fast release of the drug could be due to several reasons: alendronate that could be adsorbed in the outer surface of the matrix or by the existent alendronate gradient between mesoporous matrix and delivery medium. Therefore, after 24 h of assay, $28 \%$ of the total amount of alendronate adsorbed was delivered from MCM-41- $\mathrm{NH}_{2}$, whereas at the same time this percentage was $58 \%$ for unmodified MCM-41 (Table 2). On the other hand, $11 \%$ of the total alendronate loaded was released after $24 \mathrm{~h}$ of assay from SBA-15- $\mathrm{NH}_{2}$ matrix, whereas $55 \%$ of alendronate loaded was delivered after this time from SBA-15. After such burst effect, the alendronate was released to the medium in a sustained manner following first order kinetics for unmodified and modified MCM-41 materials and zero order or linear kinetics for unmodified and modified SBA-15 materials. Moreover, the increase in the total drug delivery time in functionalized materials compared with unmodified matrices (Table 2) can be ascribed to the stronger interactions between phosphonate groups from alendronate and amino groups covering the pore walls. This interaction led to a decrease in the alendronate delivery rate. This work evidences that the amount of alendronate adsorbed and drug 
Table 2 Alendronate loading and release data from MCM-4I and SBA-I 5 before and after functionalization with amino groups

\begin{tabular}{|c|c|c|c|c|}
\hline Material & $\begin{array}{l}\text { Surface area, } \\
S_{\mathrm{BET}}\left(\mathrm{m}^{2} / \mathrm{g}\right)\end{array}$ & $\begin{array}{l}\text { Alendronate } \\
\text { loaded (\%) }\end{array}$ & $\begin{array}{l}\text { Alendronate released } \\
\text { after } 24 \mathrm{~h}(\%)\end{array}$ & $\begin{array}{l}\text { Total delivery } \\
\text { time (h) }\end{array}$ \\
\hline MCM-4I & 1157 & 14 & 58 & 72 \\
\hline MCM-4I-NH & 782 & 37 & 28 & $264^{a}$ \\
\hline SBA- 15 & 719 & 8 & 55 & 264 \\
\hline SBA-15-NH ${ }_{2}$ & 385 & 22 & II & $264^{b}$ \\
\hline
\end{tabular}

Notes: ancomplete delivery (76\%), 'ncomplete delivery (69\%).

delivery rate can be controlled by appropriately modifying the mesoporous carriers with amino groups. In this sense, organic functionalization allows a higher control over drug loading and release kinetics.

Thus, surface area was found to be an important factor in the loading capacity of these mesoporous materials. It was found that the higher the surface area, the higher the drug adsorption. This relationship was observed for both unmodified and functionalized materials.

\section{Influence of pore volume}

As previously commented, adsorption of biologically active molecules is a surface phenomenon that takes place by attracting interactions between the silanol groups in the pore walls (or functional groups in the case of functionalized materials) and functional groups of the guest molecule. Thus, the amount of molecules adsorbed will depend on the pore diameter and surface area as the limiting factor. However, when the confinement of really large molecules is aimed, such as large-size and large-volume proteins, pore volume seems to play a key role in molecule adsorption.

Recently, in an effort to promote the loading of large biomolecules, mesostructured cellular foams (mesocellular foams, MCFs) have been employed as host matrices for the adsorption of different enzymes and proteins (Han et al 1999; Zhang et al 2007). The synthesis of MCFs type materials is carried out by employing triblock copolymers and introducing a swelling agent, such as 1,3,5-trimethylbenzene (TMB) into the structure directing template (Schmidt-Winkel et al 1999). TEM images of MCF compared to MCM-41 and
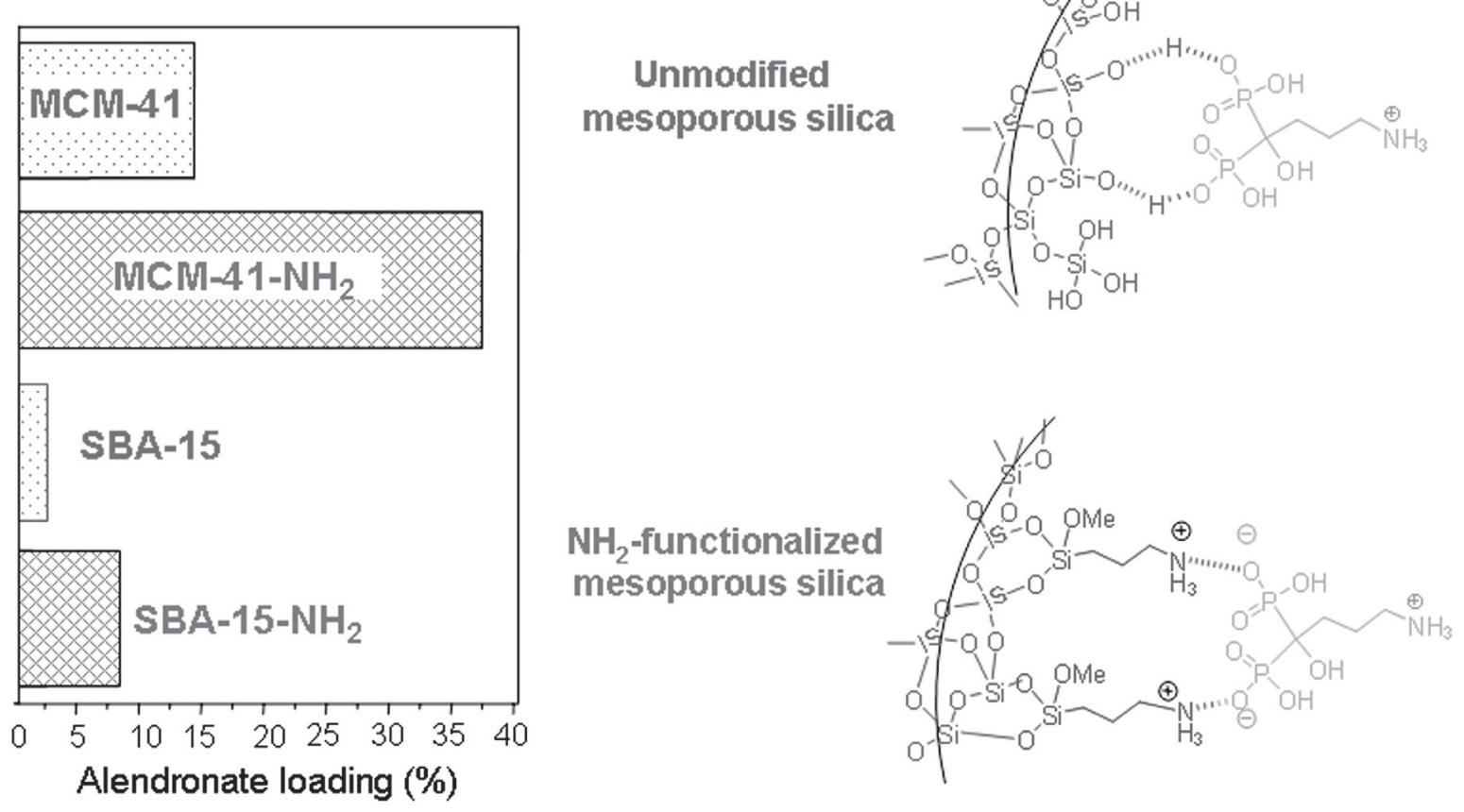

Figure 5 Alendronate adsorption on MCM-4I and SBA- 15 mesoporous materials before and after functionalization with amino groups. 
SBA-15 mesoporous materials are displayed in Figure 6. The characteristic two-dimensional hexagonal arrays of pores of MCM-41 and SBA-15 mesoporous matrices can be observed. Moreover, MCF exhibits three-dimensional, continuous, ultra-large pore mesoporous structures with large spherical cells interconnected by uniform windows (see arrows in Figure 6). $\mathrm{N}_{2}$ adsorption measurements revealed that MCM-41 and SBA-15 mesoporous materials exhibit type IV isotherms, typical of ordered mesoporous materials. The shape of the hysteresis loops points to cylindrical mesopores with very narrow pore size distributions (Figure 6) (Gregg et al 1982). In the case of MCFs, the sharp rise in the adsorption/desorption isotherms at relative pressures close to 1 points to the existence of large mesopores in these materials (Gregg et al 1982). Pore size distributions of different mesoporous matrices are also shown in Figure 6. The pore diameter of MCM-41 and SBA-15 materials are ca 3 and $9 \mathrm{~nm}$, respectively. MCF presents spherical cells of ca $28 \mathrm{~nm}$ with windows of ca 18 . The diameter of spherical cells and windows can be modulated by adjusting the amount of swelling agents and the synthesis temperature (SchmidtWinkel et al 1999). When the confinement of large-size BSA is targeted, mesoporous matrices exhibiting large pore diameters are needed. Thus, SBA-15 and MCF before and after functionalization using amino groups where tested as delivery systems for BSA. The amount of BSA loaded in MCF materials was higher (24\%) than in SBA-15 matrices (15\%), due to the higher pore volume in the former (Table 3 ). It should be highlighted that the surface area is not the determinant factor that governs protein adsorption, because it exhibits the opposite trend to protein loading (Table 3). The amount of BSA loaded after functionalization followed the same trend that unmodified materials, ie, $\mathrm{MCF}-\mathrm{NH}_{2}$ loaded more BSA $(27 \%)$ than SBA-15- $\mathrm{NH}_{2}(10 \%)$, as a result of the higher pore volume of the former. The increase in BSA loading after functionalization of MCF matrix can

\section{TEM images}

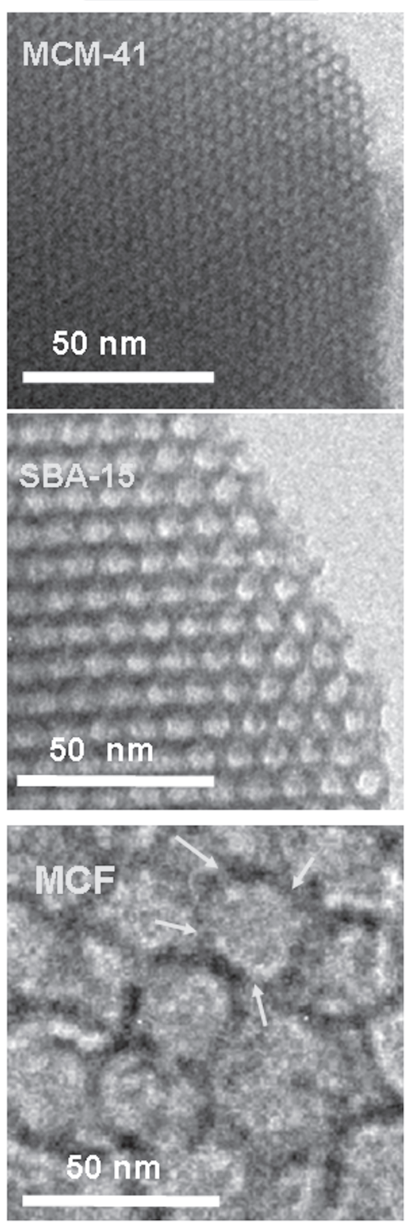

\section{$\mathrm{N}_{2}$ Adsorption}
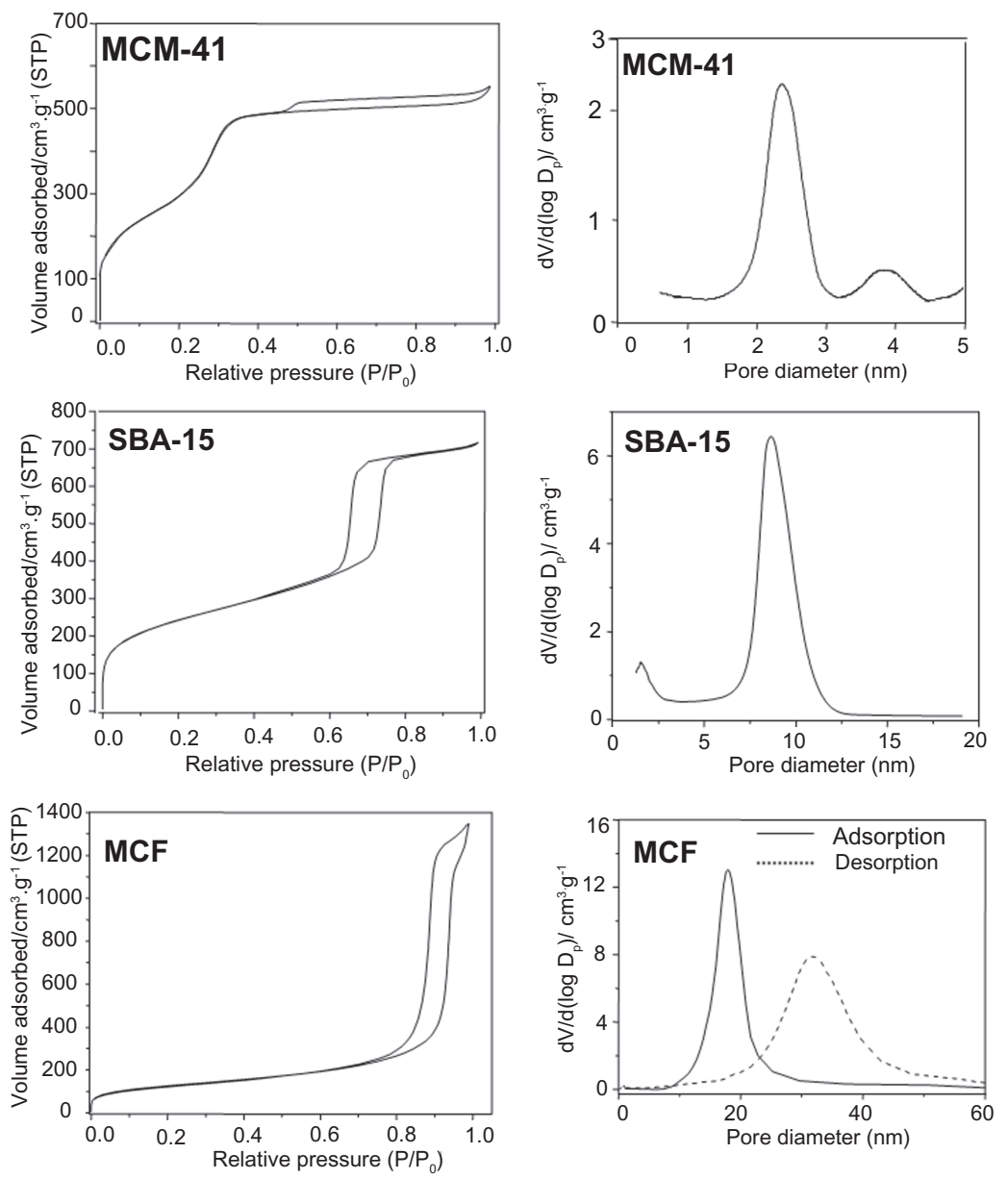

Figure 6 TEM images of MCM-4I, SBA-15, and MCF mesoporous materials. Their corresponding nitrogen adsorption isotherms and pore size distributions are also displayed. 
Table 3 BSA loading and release data from SBA-I5 and MCF materials before and after functionalization with amino groups

\begin{tabular}{lllllll}
\hline Material & $\begin{array}{l}\text { Pore diameter } \\
(\mathbf{n m})\end{array}$ & $\begin{array}{l}\text { Surface area, } \\
\mathbf{S}_{\mathrm{BET}}\left(\mathbf{m}^{2} / \mathbf{g}\right)\end{array}$ & $\begin{array}{l}\text { Pore volume } \\
\left(\mathbf{c m}^{3} / \mathbf{g}\right)\end{array}$ & $\begin{array}{l}\text { BSA loaded } \\
(\%)\end{array}$ & $\begin{array}{l}\text { BSA released } \\
\text { after 24 h (\%) }\end{array}$ & $\begin{array}{l}\text { Total \% of BSA } \\
\text { released after } \\
\mathbf{2 6 4} \mathbf{h} \text { of assay }\end{array}$ \\
\hline SBA-15 & 8.5 & 874 & 1.1 & 20 & 74 & 93 \\
SBA-15-NH 2 & 6.9 & 348 & 0.7 & 21 & 27 & 87 \\
MCF & $17.9^{\mathrm{a}}$ & 452 & 1.9 & 24 & 62 & 90 \\
MCF-NH & $15.7^{\mathrm{a}}$ & 275 & 1.6 & 27 & 22 & 86 \\
\hline
\end{tabular}

Notes: ${ }^{a}$ The pore diameter corresponds to the size of the windows present in the spherical cells.

be attributed to the higher attracting electrostatic interactions of amino groups with the amide groups of protein. However, as it was previously mentioned, organic functionalization always leads to a decrease in pore diameter (Table 3 ). The pore diameter of SBA-15 $(8.5 \mathrm{~nm})$ is just on the limit of BSA size and therefore, after functionalization the pore diameter decreased to $6.9 \mathrm{~nm}$ and consequently, a decrease in the amount of BSA adsorbed was observed. On the other hand, organic functionalization with amino groups strongly influenced the BSA release kinetics. As it can be observed in Figure 7, unmodified matrices exhibited an initial burst effect

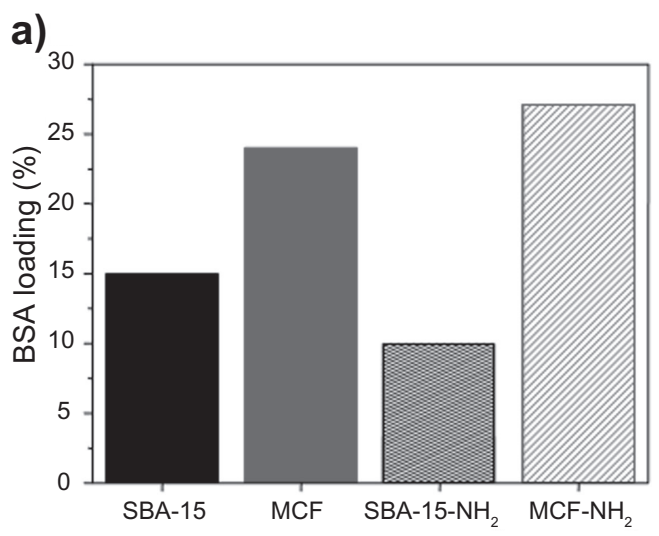

b)

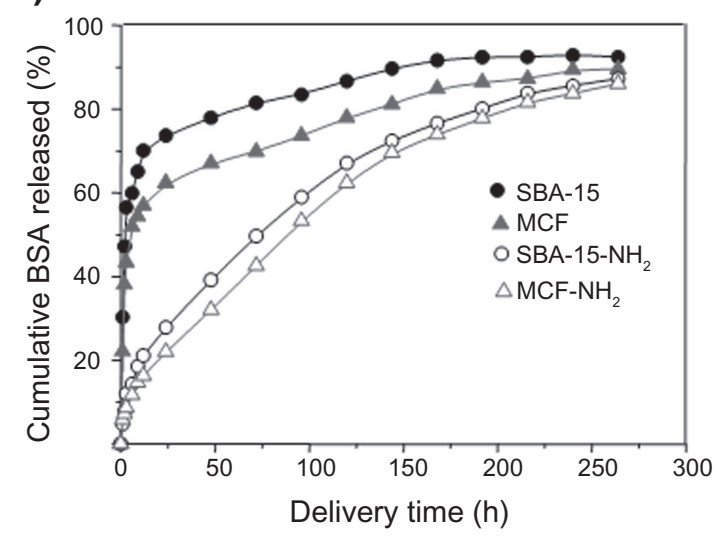

Figure 7 a) Amount of BSA loaded (\%) and b) BSA delivery profiles for SBA- 15 and MCF mesoporous matrices before and after functionalization using amino groups. when almost $60 \%$ of the protein was quickly released to the delivery medium and then the loaded protein was delivered in a controlled fashion. On the contrary, the initial burst in amino-modified matrices was drastically reduced to ca $10 \%$, and more than $80 \%$ of the loaded BSA was released to the medium in a sustained manner. After $24 \mathrm{~h}$ of assay, $74 \%$ of the total BSA loaded in SBA-15 was released to the medium, whereas after the same time only $27 \%$ was released to the medium from SBA-15- $\mathrm{NH}_{2}$ matrix. Moreover, after $24 \mathrm{~h}$ of delivery test $62 \%$ of the loaded BSA was released from unmodified MCF material and this percentage decreased to $22 \%$ after functionalization with amino groups. This research work demonstrates that BSA can be successfully adsorbed into large-volume mesoporous matrices exhibiting the appropriate pore diameter to allow protein confinement. Therefore, when the loading of large-size and large-volume biologically active molecules is targeted, pore diameter acts as the limiting factor and pore volume determines the amount of molecule hosted.

\section{Conclusions}

In this work it has been shown that available pore volume and surface play a key role in the protein loading capacity of silica-based ordered mesoporous materials. However, if the pore opening is not wide enough, access of the protein molecules to much of this surface area and pore volume will be restricted. Ideally, if large biomolecules such as certain proteins are targeted to be adsorbed in ordered mesoporous materials, these matrices should present several characteristics: pore size large enough to allow diffusion into the pores, surface area as high as possible to allow a large retention percentage, and pore volume as high as possible to offer available space into the mesopores to be filled by the protein. Recent advances in nanotechnology offer the possibility of appropriately tailoring the structural and textural properties of mesoporous silicas depending on the desired application. The outstanding features of silica-based ordered mesoporous materials open up promising expectations in the 
biomedical field because they can be employed as starting materials for the further design of scaffolds for bone tissue regeneration.

\section{Disclosure}

The authors report no conflicts of interest in this work.

\section{References}

Antochshuk V, Jaroniec M. 2000. Functionalized mesoporous materials obtained via interfacial reactions in self-assembled silica-surfactant systems. Chem Mater, 12:2496-501.

Bagshaw SA, Prouzet E, Pinnavaia TJ. 1995. Templating of mesoporous molecular-sieves by non-ionic polyethylene oxide surfactants. Science, 269:1242-4.

Balas F, Manzano M, Horcajada P, et al. 2006. Confinement and controlled release of bisphosphonates on ordered mesoporous silica-based materials. J Am Chem Soc, 128:8116-7.

Balas F, Manzano M, Colilla M, et al. 2008. L-Trp adsorption into silica mesoporous materials to promote bone formation. Acta Biomater, 4:514-22.

Beck JS, Vartuli JC, Roth WJ, et al. 1992. A new family of mesoporous molecular-sieves prepared with liquid-crystal templates. J Am Chem Soc, 114:10834-43.

Carter DC, Ho JX. 1994. Structure of serum albumin. Adv Protein Chem, 45:153-203.

Cavallaro G, Pierro P, Palumbo FS, et al. 2004. Drug delivery devices based on mesoporous silicate. Drug Delivery, 11:41-6.

Che S, García-Bennett AE, Yokoi T, et al. 2003. Mesoporous silica of novel structures with periodic modulations synthesised by anionic surfactant templating route. Nature Materials, 2:801-5.

Chen Q, Miyaji F, Kokubo T, et al. 1999. Apatite formation on PDMS-modified $\mathrm{CaO}-\mathrm{SiO}_{2}-\mathrm{TiO}_{2}$ hybrids prepared by sol-gel process. Biomaterials, 20:1127-32.

Cheng YY, Lin SH, Chang HC, et al. 2003. Probing adsorption, orientation and conformational changes of cytochrome $\mathrm{c}$ on fused silica surfaces with the soret band. J Phys Chem A, 107:10687-94.

Colilla M, Salinas AJ, Vallet-Regí M. 2006. Amino-polysiloxane hybrid materials for bone reconstruction. Chem Mater, 18:5676-83.

Deere J, Magner E, Wall JG, et al. 2002. Mechanistic and structural features of protein adsorption onto mesoporous silicates. J Phys Chem B, 106:7340-7.

Doadrio JC, Sousa EMB, Izquierdo-Barba I, et al. 2006. Functionalization of mesoporous materials with long alkyl chains as a strategy for controlling drug delivery pattern. $J$ Mater Chem, 16:462-7.

Gregg SJ, Sing KSW. 1982. Adsorption, surface area and porosity. 2nd ed. New York: Academic Press.

Firouzi A, Atef F, Oertli AG, et al. 1997. Alkaline lyotropic silicatesurfactant liquid crystals. J Am Chem Soc, 119:3596-610.

Han YJ, Stucky GD, Butler A. 1999. Mesoporous silicate sequestration and release of proteins. J Am Chem Soc, 121:9897-8.

Hartmann M. 2005. Ordered mesoporous materials for bioadsorption and biocatalysis. Chem Mater, 17:4577-93.

Hench LL, Wilson J. 1984. Surface active biomaterials. Science, 226:630-6.

Hench LL. 1998. Bioceramics. J Am Ceram Soc, 81:1705-28.

Hoffmann F, Cornelius M, Morell J, et al. 2006. Silica-based mesoporous organic-inorganic hybrid materials. Angew Chem Int Ed, 45:3216-51.

Horcajada P, Rámila A, Pérez-Pariente J, et al. 2004. Influence of pore size of MCM-41 matrices on drug delivery rate. Microporous Mesoporous Mater, 68:105-9.

Hulbert SF. 1993. The use of alumina and zirconia in surgical implants. In: Hench LL, Wilson J (eds). An Introduction to Bioceramics. Singapore: World Scientific, pp. 25-40.
Inagaki S, Fukushima Y, Kuroda K. 1993. Synthesis of highly ordered mesoporous materials from a layered polysilicate. Chem Comm, 8:680-2.

Inagaki S, Koiwai A, Suzuki N, et al. 1996. Syntheses of highly ordered mesoporous materials, FSM-16, derived from kanemite. Bull Chem Soc Jpn, 69:1449-57.

Izquierdo-Barba I, Ruiz-González L, Doadrio JC, et al. 2005a. Tissue regeneration. A new property of mesoporous materials. Solid State Sci, 7:983-9.

Izquierdo-Barba I, Martínez A, Doadrio AL, et al. 2005b. Release evaluation of drugs from ordered three-dimensional silica structures. Eur J Pharm Sci, 26:365-73.

Izquierdo-Barba I, Manzano M, Colilla M, et al. 2008. Silica-based ordered mesoporous materials for biomedical applications. Key Eng Mater, $377: 133-50$

Kaneda M, Tsubakiyama T, Carlsson A, et al. 2002. Structural study of mesoporous MCM-48 and carbon networks synthesised in the spaces of MCM-48 by electron crystallography. $J$ Phys Chem B, 106:1256-66.

Katiyar A, Ji L, Smirniotis PG, et al. 2005. Adsorption of bovine serum albumin and lysozyme on siliceous MCM-41. Microporous Mesoporous Mater, 80:311-20.

Kokubo T, Kushitani H, Sakka S, et al. 1990. Solutions able to reproduce in vivo surface-structure changes in bioactive glass-ceramic A-W3. J Biomed Mater Res, 24:721-34.

Kresge CT, Leonowicz ME, Roth WJ, et al. 1992. Ordered mesoporous molecular sieves synthesised by a liquid-crystal template mechanism. Nature, 359:710-12.

Kruk M, Jaroniec M, Sakamoto Y, et al. 2001. Determination of pore size and pore wall structure of MCM-41 by using nitrogen adsorption, transmission electron microscopy, and X-ray diffraction. J Phys Chem $B, 104: 292-301$.

Langer R, Vacanti JP. 1993. Tissue engineering. Science, 260:920-6.

Lee $\mathrm{CH}$, Mou CY, Ke SC, et al. 2006. Effect of spin configuration on the reactivity of cytochrome c immobilized in mesoporous silica. Mol Phys, 104:1635-41.

Lei J, Fan J, Yu C, et al. 2004. Immobilization of enzymes in mesoporous materials: controlling the entrance to nanospace. Microporous Mesoporous Mater, 73:121-8.

Liu J, Fena X, Fryxell GE, et al. 1998. Hybrid mesoporous materials with functionalised monolayers. Adv Mater, 10:161-4.

Manzano M, Arcos D, Rodríguez-Delgado M, et al. 2006. Bioactive star gels. Chem Mater, 18:5696-703.

Manzano M, Aina V, Areán CO, et al. 2008. Studies on MCM-41 mesoporous silica for drug delivery: Effect of particle morphology and amine functionalization. Chem Eng $J, 137: 31-7$.

Muñoz B, Ramila A, Pérez-Pariente J, et al. 2003. MCM-41 organic modification as drug delivery rate regulator. Chem Mater, 15:500-3.

Nieto A, Balas F, Colilla M, et al. 2008. Functionalization degree of SBA-15 as key factor to modulate sodium alendronate dosage. Microporous and Mesoporous Mater, doi: 10.1016/j.micromeso.2008.03.025.

Peters T. 1995. All about albumin: biochemistry, genetics and medical applications. San Diego: Academy Press.

Qu F, Zhu G, Huang S, et al. 2006. Controlled release of captopril by regulating the pore size and morphology of ordered mesoporous silica. Microporous Mesoporous Mater, 92:1-9.

Ravikovitch PI, Neimark AV. 2002a. Density functional theory of adsorption in spherical cavities and pore size characterisation of templated nanoporous silicas with cubic and three-dimensional hexagonal structures. Langmuir, 18:1550-60.

Ravikovitch PI, Neimark AV. 2002b. Experimental confirmation of different mechanisms of evaporation of ink-bottle type pores: equilibrium, pore blocking, and cavitation. Langmuir, 18:9830-7.

[RCSB] Research Collaboratory for Structural Bioinformatics. 2008. Protein Data Bank [online]. Accessed September 1, 2008. URL: http:// www.pdb.org.

Ryoo R, Kim JM, Ko CH, et al. 1996. Disordered molecular sieve with branched mesoporous channel network. J Phys Chem B, 100:17718-21. 
Sanchez C, Julián B, Belleville P, et al. 2005. Applications of hybrid organic-inorganic nanocomposites. J Mater Chem, 5:3559-92.

Schmidt-Winkel P, Lukens WW, Zhao D, et al. 1999. Mesocellular siliceous foams with uniformly sized cells and windows. J Am Chem Soc, 121:254-5.

Slowing II, Trewyn BG, Lin VS-Y. 2007. Meoporous silica nanoparticles for intracellular delivery of membrane-impermeable proteins. $J \mathrm{Am}$ Chem Soc, 129:8845-9.

Song S-W, Hidajat K, Kawi S. 2005. Functionalized SBA-15 materials as carriers for controlled drug delivery: influence of surface properties on matrix-drug interactions. Langmuir, 21:9568-75.

Sugio S, Kashima A, Mochizuki S, et al. 1999. Crystal structure of human serum albumin at 2.5 angstrom resolution. Protein Eng, 12:439-46.

Tsuru K, Ohtsuki C, Osaka A, et al. 1997. Bioactivity of sol-gel derived organically modified silicates.1. In vitro examination. J Mater Sci Mater Med, 8:157-61.

Vallet-Regí M. 2001a. Ceramics for medical applications. Perspective Article. J Chem Soc Dalton Trans, 2:97-108.

Vallet-Regí M, Rámila A, Del Real RP, et al. 2001b. A new property of MCM-41: Drug delivery system. Chem Mater, 13:308-11.

Vallet-Regí M, González-Calbet JM. 2004a. Calcium phosphates as substitution of bone tissues. Prog Solid State Sci, 32:1-31.

Vallet-Regí M, Doadrio JC, Doadrio AL, et al. 2004b. Hexagonal ordered mesoporous material as a matrix for the controlled release of amoxicillin. Solid State Ionics, 172:435-9.

Vallet-Regí M, Izquierdo-Barba I, Rámila A, et al. 2005. Phosphorous doped MCM-41 as bioactive material. Solid State Sci, 7:233-7.

Vallet-Regí M, Arcos D. 2006a. Nanostructured Hybrid materials for bone tissue regeneration. Current Nanosci, 2:179-89.

Vallet-Regí M, Salinas AJ, Arcos D. 2006b. From bioactive glasses to star gels. J Mater Sci Mater Med, 7:1011-7.

Vallet-Regí M, Ruiz-González L, Izquierdo-Barba I, et al. 2006c. Revisiting silica based ordered mesoporous materials: medical applications. J Mater Chem, 16:26-31.

Vallet-Regí M. 2006d. Bone repair and regeneration possibilities. Mater-wiss $u$ Werkstofftech, 37:478-84.

Vallet-Regí M, 2006e. Ordered mesoporous materials in the context of drug delivery systems and bone tissue engineering. Chem Eur J, 12:5934-43.

Vallet-Regí M, Balas F, Arcos D. 2007a. Mesoporous materials for drug delivery. Angew Chem Int Ed, 46:7548-58.

Vallet-Regí M, Balas F, Colilla M, et al. 2007b. Bone-regenerative bioceramic implants with drug and protein controlled delivery capability. Prog Solid State Chem, doi:10.1016/j.progsolidstchem. 2007.10.002.
Vallet-Regí M, Colilla M, Izquierdo-Barba I. 2008. Bioactive mesoporous silicas as controlled delivery systems: application in bone tissue regeneration. J Biomed Nanotechnol, 4:1-15.

Vinu A, Murugesan V, Tangermann O, et al. 2004. Adsorption of cytochrome $\mathrm{c}$ on mesoporous molecular sieves: Influence of $\mathrm{pH}$, pore diameter, and aluminium incorporation. Chem Mater, 16:3056-65.

Wise DL, Trantolo DJ, Altobelli DE, et al. 1995. Encyclopedic handbook of biomaterials and bioengineering. New York: Marcel Dekker.

Xue JM, Shi M. 2004. PLGA/mesoporous silica hybrid structure for controlled drug release. J Control Release, 98:209-17.

Yamamuro Y, Hench LL, Wilson J. 1990a. Calcium phosphate and hydroxyapatite ceramics. In Handbook of bioactive ceramics, vol. 2. Boca Raton: CRC Press.

Yamamuro Y, Hench LL, Wilson J. 1990b. Bioactive glasses and glass ceramics. In Handbook of bioactive ceramics, vol. 1. Boca Raton: CRC Press

Yanagisawa T, Shimizu T, Kuroda K, et al. 1990. The preparation of alkyltrimethylammonium-kanemite complexes and their conversion to microporous materials. Bull Chem Soc Jpn, 63:988-92.

Yang Q, Wang SH, Fan PW, et al. 2005. pH-responsive carrier system based on carboxylic acid modified mesoporous silica and polyelectrolyte for drug delivery. Chem Mater, 17:5999-6003.

Yiu HHP, Wright PA, Botting NP. 2001a. Enzyme immobilisation using SBA-15 mesoporous molecular sieves with functionalised surfaces. J Mol Catal B: Enzym, 15:81-92.

Yiu HHP, Botting CH, Botting NP, et al. 2001b. Size selective protein adsorption on thiol-functionalised SBA-15 mesoporous molecular sieve. Phys Chem Chem Phys, 3:2983-5.

Yiu HHP, Wright PA. 2005. Enzymes supported on ordered mesoporous solids: a special case of an inorganic-organic hybrid. J Mater Chem, 15:3690-700.

Zeng W, Qian XF, Zhang YB, et al. 2005. Organic modified mesoporous MCM-41 through solvothermal process as drug delivery system. Mater Res Bull, 40:766-72.

Zhang J, Luz Z, Goldfarb D. 1997. EPR studies of the formation mechanism of the mesoporous material MCM-41 and MCM-50. J Phys Chem B, 101:7087-94.

Zhang X, Guan RF, Wu DQ, et al. 2007. Preparation of amino-functionalized mesostructured cellular foams and application as hosts for large biomolecules. J Mater Sci Mater Med, 18:877-82.

Zhao DY, Feng JL, Huo QS, et al. 1998. Triblock copolymer syntheses of mesoporous silica with periodic 50 to 300 angstrom pores. Science, 279:548-52.

Zhu YF., Shi JL, Shen WH, et al. 2005. Stimuli-responsive controlled drug release from a hollow mesoporous silica sphere/polyelectrolyte multilayer core-shell structure. Angew Chem Int Ed, 44:5083-7. 